NOTA DE PESQUISA

\title{
PROPOSTA PARA TESTE CARRAPATICIDA POR IMERSÃO DE LARVAS DE Rhipicephalus (Boophilus) microplus: AVALIAÇÃO EM CIPERMETRINA E AMITRAZ
}

\author{
ANTONIO P. DE SOUZA ${ }^{1}$, LUANA PAULA H. N. VEIGA ${ }^{2}$, VALDOMIRO BELLATO ${ }^{1}$, AMÉLIA APARECIDA SARTOR ${ }^{1}$, \\ CRISTINA P. CARDOSO ${ }^{1}$, ANA PAULA DE O. NUNES ${ }^{3}$,
}

\begin{abstract}
SOUZA, A.P.; VEIGA, L.P.H.N.; BELLATO, V.; SARTOR, A.A.; CARDOSO, C.P.; NUNES, A.P.O. [Proposal of an acaricide laboratory test larvae immersion of Rhipicephalus (Boophilus) microplus: evaluation with cypermethrin and amitraz]. Proposta para teste carrapaticida por imersão de larvas de Rhipicephalus (Boophilus) microplus: avaliação em cipermetrina e amitraz. Revista Brasileira de Parasitologia Veterinaria, v. 17, n. 4, p. 242-245, 2008. Universidade do Estado de Santa Catarina, Centro de Ciências Agroveterinárias, Avenida Luis de Camões, 2090, Bairro Conta Dinheiro, Lages, SC 88520-000, Brazil. E-mail: a2aps@cav.udesc.br

The aim of this work was to develop a technique of acaricides evaluation by immersion of larvae. Larvae were obtained from 30 engorged females of Riphicephalus (Boophilus) microplus were collected in a ranch in Lages, Santa Catarina state, Brazil. Larvae were obtained from $10 \mathrm{mg}$ of eggs placed into $5 \mathrm{ml}$ disposable syringes, previously prepared and kept under controlled conditions of laboratory, and collected between the third and the twelfth day of oviposition. Seven to ten days old larvae were submitted to the acaricide laboratory test, in ten replications, by their immersion in eight dilutions of commercial acaricides (amitraz or cypermethrin), for 30 seconds; for each replication and commercial product, a control group was immersed in distilled water. Results were evaluated 24 hours after each treatment by counting the number of live and dead larvae. The mean $\mathrm{LD}_{50}$ values and standard error of the mean for cypermethrin and amitraz were $2.99 \pm 0.36$ and $17.22 \pm 4.20 \mathrm{ppm}$, respectively, for a confidence interval of $95 \%$. The proposed acaricide laboratory test procedures by larvae immersion offered promising results for the evaluation of acaricides.
\end{abstract}

KEY WORDS: Ticks, in vitro test, acaricide.

\section{RESUMO}

Objetivou-se desenvolver uma técnica de avaliação de carrapaticidas por imersão de larvas de Riphicephalus (Boophilus) microplus. As larvas foram obtidas a partir de ovos de 30 teleóginas coletadas em bovinos de uma propriedade rural do município de Lages, SC, Brasil, os quais foram acondicionados em alíquotas de $10 \mathrm{mg}$ por seringa descartável de $5 \mathrm{ml}$, previamente preparadas e mantidas em câmara climatizada

\footnotetext{
${ }^{1}$ Departamento de Medicina Veterinária, Centro de Ciências Agroveterinárias (CAV), Universidade do Estado de Santa Catarina (UDESC), Avenida Luiz de Camões, 2090, Bairro Conta Dinheiro, Lages, SC 88520-000, Brasil. E-mail a2aps@cav.udesc.br

${ }^{2}$ Curso de Pós-Graduação em Ciência Animal, CAV, UDESC, Lages, SC.

${ }^{3}$ Curso de Medicina Veterinária, CAV, UDESC, Lages, SC. Bolsista de I C- PIBIC(CNPq/ UDESC).
}

até a eclosão. Larvas com sete a dez dias de idade foram submetidas à imersão por 30 segundos em oito diluições de produtos carrapaticidas comerciais à base de amitraz e de cipermetrina, cada uma com dez repetições, sendo mantido um grupo controle para cada repetição e produto, o qual foi imerso em água destilada. A leitura foi realizada 24 horas após o tratamento com a contagem de larvas vivas e mortas. A média de $\mathrm{DL}_{50}$ para cipermetrina foi de 2,99ppm e o erro padrão de 0,36 e para o amitraz foi de 17,22ppm e o erro padrão de 4,20, com intervalo de confiança (IC) $95 \%$.

PALAVRAS-CHAVE: Carrapatos, teste in vitro carrapaticida.

Reconhecidamente, o problema da resistência do Rhipicephalus (Boophilus) microplus aos carrapaticidas vem aumentando consideravelmente em todas as regiões onde ele 
está presente e que utilizam produtos químicos como principal recurso para o seu controle. Este fato pode ser observado in vivo, verificando-se o efeito dos compostos acaricidas sobre os carrapatos no hospedeiro, ou em laboratório, por meio da imersão in vitro de fêmeas ingurgitadas e larvas não alimentadas. Sabatini et al. (2001) citaram o teste de imersão de larvas (TIL) (SHAW, 1966) e o teste do pacote de larvas (TPL) (STONE; HAYDOCK, 1962) como métodos utilizados para detecção da resistência, sendo somente o TLP adotado pela Organização das Nações Unidas para Agricultura e Alimentação (FAO).

O monitoramento das populações de carrapatos, em relação aos carrapaticidas por meio de testes in vitro, é um procedimento essencial na detecção precoce de problemas de resistência tendo em vista que o uso de acaricidas ainda se constitui no principal instrumento de controle do carrapato bovino R. (B.) microplus (VARGAS et al., 2003). Em função disso, os pesquisadores têm se preocupado em aprimorar as técnicas de diagnóstico da resistência. Exemplos destas novas metodologias é a modificação da técnica do pacote de larvas (LPT), publicada por Miller et al. (2002) e por Barci e Nogueira (2006).

Miller et al. (2002) descreveram uma modificação da técnica do teste dos envelopes preparados com papéis impregnados com acaricidas (TPL) para quantificar a suscetibilidade do $R$. (B.) microplus ao amitraz.

A realização do presente trabalho teve como objetivo desenvolver uma técnica de avaliação de carrapaticidas por imersão de larvas.

Foram utilizadas 30 teleóginas de $R$. (B.) microplus coletadas de bovinos em uma propriedade rural do município de Lages, SC, , as quais foram colocadas em placas de Petri em câmara climatizada regulada à temperatura de $27 \pm 1^{\circ} \mathrm{C}$ e $80 \pm$ $10 \%$ de UR para realizarem postura. Para obtenção das larvas, foram utilizadas as posturas do terceiro ao décimo segundo dia, após a incubação. Foram acondicionados 10mg de ovos por seringa plástica, descartável de $5 \mathrm{ml}$, previamente preparada, pelo corte da extremidade próxima do canhão e feito um orifício de aproximadamente $1 \mathrm{~mm}$ de diâmetro, na metade desta. O êmbolo foi posicionado de forma a fechar o orifício, sendo que a extremidade cortada foi fechada com tecido de malha fina (voal), fixado com elástico ortodôntico de diâmetro de 7,94mm, para impedir a fuga das larvas. As seringas foram mantidas na câmara climatizada, nas condições de temperatura e umidade anteriormente descritas, até a realização dos testes.

Larvas, resultantes das incubações, com idade entre sete e 10 dias foram submetidas à imersão em carrapaticidas comerciais à base de amitraz ${ }^{4}(12,5 \%)$ à 250,0, 125,0, 62,5, 31,25, $15,62,7,85,3,9$, e 1,95 ppm e à base de cipermetrina ${ }^{5}$ (15\%), à $150,0,75,0,37,5,18,75,9,37,4,68,2,34$ e 1,17ppm. Foram realizados 10 testes, sendo mantido um grupo controle para

\footnotetext{
${ }^{4}$ Triatox®, Schering-Plough.

${ }^{5}$ Ectic ${ }^{\circledR}$, Tortuga.
}

cada repetição e produto, o qual foi imerso em água destilada. Cada grupo de larvas foi imerso nas respectivas diluições, e o grupo controle, em água destilada, durante 30 segundos, posicionando o êmbolo posteriormente ao orifício para permitir a saída de ar e consequentemente o preenchimento total da seringa com o produto a testar. Após a imersão, a extremidade fechada com o tecido de trama fina, foi colocada em contato com papel absorvente para eliminar o líquido excedente. O embolo foi novamente posicionado de forma a fechar o orifício e cada seringa foi colocada em suporte, com a extremidade cortada e vedada, para cima, e novamente colocada na câmara climatizada por 24 horas. Posteriormente, foi pressionado o embolo da seringa até a margem cortada e, as larvas retiradas com o auxílio de um pincel fino, sobre uma folha de papel branco. A leitura foi realizada, com o auxílio de uma fonte luminosa, utilizando-se uma lâmpada de 40 Watts, contando-se as larvas vivas e mortas.

A seqüência da técnica de imersão de larvas está representada na Figura 1.

O cálculo da $\mathrm{DL}_{50}$ foi realizado a partir da linha de regressão, utilizando-se o programa "Sigma plot". Foi calculado a média da eficácia dos carrapaticidas nas diferentes concentrações, o desvio-padrão e o erro padrão.

Nas Tabelas 1 e 2 podem ser observados os resultados do percentuais de mortalidade das larvas no teste de imersão em

Tabela 1. Percentuais de mortalidade de larvas de Rhipicephalus (Boophilus) microplus, no teste de imersão, utilizando-se acaricida à base de cipermetrina e $\mathrm{DL}_{50}$.

\begin{tabular}{|c|c|c|c|c|c|c|c|c|c|c|}
\hline \multirow{2}{*}{$\begin{array}{l}\text { Repe- } \\
\text { tições }\end{array}$} & \multirow{2}{*}{$\begin{array}{l}\text { Con- } \\
\text { trole }\end{array}$} & \multicolumn{9}{|c|}{ Concentrações (ppm) } \\
\hline & & 150 & 75 & 37,5 & 18,75 & 9,37 & 4,68 & 2,34 & 1,17 & $\mathrm{DL}_{50}$ \\
\hline 1 & 0,0 & 96,0 & 93,7 & 63,7 & 83,5 & 76 & 38,9 & 44,4 & 34,2 & 3,1 \\
\hline 2 & 0,0 & 95,1 & 87,1 & 89,6 & 68,5 & 70,5 & 47,4 & 21,1 & 22,9 & 5,3 \\
\hline 3 & 0,0 & 89,7 & 95,3 & 87,3 & 94,9 & 92,6 & 79,4 & 45,2 & 45,2 & 1,2 \\
\hline 4 & 0,0 & 99,4 & 98,8 & 96,5 & 93,7 & 87,3 & 79,0 & 24,1 & 43,1 & 2,6 \\
\hline 5 & 0,0 & 96,3 & 95,8 & 92,0 & 86,2 & 76,4 & 61,8 & 37,8 & 36,7 & 2,6 \\
\hline 6 & 0,0 & 97,3 & 93,5 & 89,7 & 86,8 & 74,3 & 51,8 & 46,5 & 37,3 & 2,6 \\
\hline 7 & 0,0 & 96,6 & 93,7 & 86,1 & 80,3 & 94,2 & 94,3 & 27,1 & 3,7 & 3,4 \\
\hline 8 & 0,0 & 95,6 & 90,6 & 84,8 & 96,4 & 91,7 & 38,3 & 46,2 & 4,9 & 4,4 \\
\hline 9 & 0,0 & 98,9 & 99,5 & 100 & 95,8 & 98,9 & 55,9 & 35,2 & 23,7 & 2,4 \\
\hline 10 & 0,0 & 97,9 & 100,0 & 97,1 & 97,4 & 98,2 & 67,1 & 27,5 & 24,5 & 2,4 \\
\hline
\end{tabular}

Tabela 2. Percentuais de mortalidade de larvas de Rhipicephalus (Boophilus) microplus, no teste de imersão, utilizando-se acaricida à base de amitraz e $\mathrm{DL}_{50}$.

\begin{tabular}{|c|c|c|c|c|c|c|c|c|c|c|}
\hline \multirow{2}{*}{$\begin{array}{l}\text { Repe- } \\
\text { tições }\end{array}$} & \multirow{2}{*}{$\begin{array}{l}\text { Con- } \\
\text { trole }\end{array}$} & \multicolumn{9}{|c|}{ Concentrações (ppm) } \\
\hline & & 250 & 125 & 52,5 & 31, & 15,62 & 7,81 & 3,90 & 1,95 & $\mathrm{DL}_{50}$ \\
\hline 1 & 0,0 & 90,1 & 82,8 & 76,3 & 93,5 & 43,8 & 46,5 & 45,2 & 8,1 & 10,1 \\
\hline 2 & 0,0 & 83,7 & 92,9 & 76,9 & 64,3 & 64,4 & 88,1 & 41,4 & 28,7 & 3,5 \\
\hline 3 & 0,0 & 89,6 & 93,4 & 93,9 & 63,5 & 58,3 & 48,9 & 41,6 & 34,5 & 6,2 \\
\hline 4 & 0,0 & 91,4 & 77,1 & 81,7 & 76,9 & 90,9 & 44,0 & 47,9 & 40,2 & 3,2 \\
\hline 5 & 0,0 & 88,3 & 81,9 & 58,9 & 48,9 & 39,2 & 35,3 & 40,7 & 24,8 & 16,5 \\
\hline 6 & 0,0 & 93,7 & 81,6 & 61,8 & 48,7 & 42,6 & 39,1 & 32,8 & 16,1 & 16,5 \\
\hline 7 & 0,0 & 73,8 & 52,9 & 65,8 & 63,6 & 62,4 & 28,8 & 25,0 & 15,6 & 24,3 \\
\hline 8 & 0,0 & 76,2 & 68,1 & 68,4 & 67,0 & 56,4 & 17,3 & 27,9 & 19,0 & 22,0 \\
\hline 9 & 0,0 & 72,0 & 57,7 & 16,2 & 61,4 & 38,3 & 48,4 & 47,8 & 19,0 & 47,9 \\
\hline 10 & 0,0 & 72,7 & 82,8 & 70,3 & 95,5 & 6,5 & 0,0 & 14,2 & 18,3 & 22,0 \\
\hline
\end{tabular}


cipermetrina e em amitraz, respectivamente, e as doses letais $_{50}$.

A média da $\mathrm{DL}_{50}$ para a cipermetrina foi de 3,0ppm com uma amplitude de variação de 1,2ppm a 5,3ppm e erro padrão médio de 0,36. Verificou-se que em alguns casos os resultados não corresponderam ao esperado, ou seja, na ordem de-

Figura 1. Seqüência da técnica de imersão de larvas de Rhipicephalus (Boophilus) microplus, (a, Postura de teleóginas de Riphicephalus (Boophilus) microplus; b, Seringas descartáveis preparadas para o teste de imersão de larvas; c, Preparação das diluições dos carrapaticidas; d, Imersão das seringas em diluições de produtos carrapaticidas; e, Retirada do excesso de produto em papel absorvente; $f$, Seringas após a imersão em diluições de produtos carrapaticidas e g, Leitura do teste de imersão de larvas).
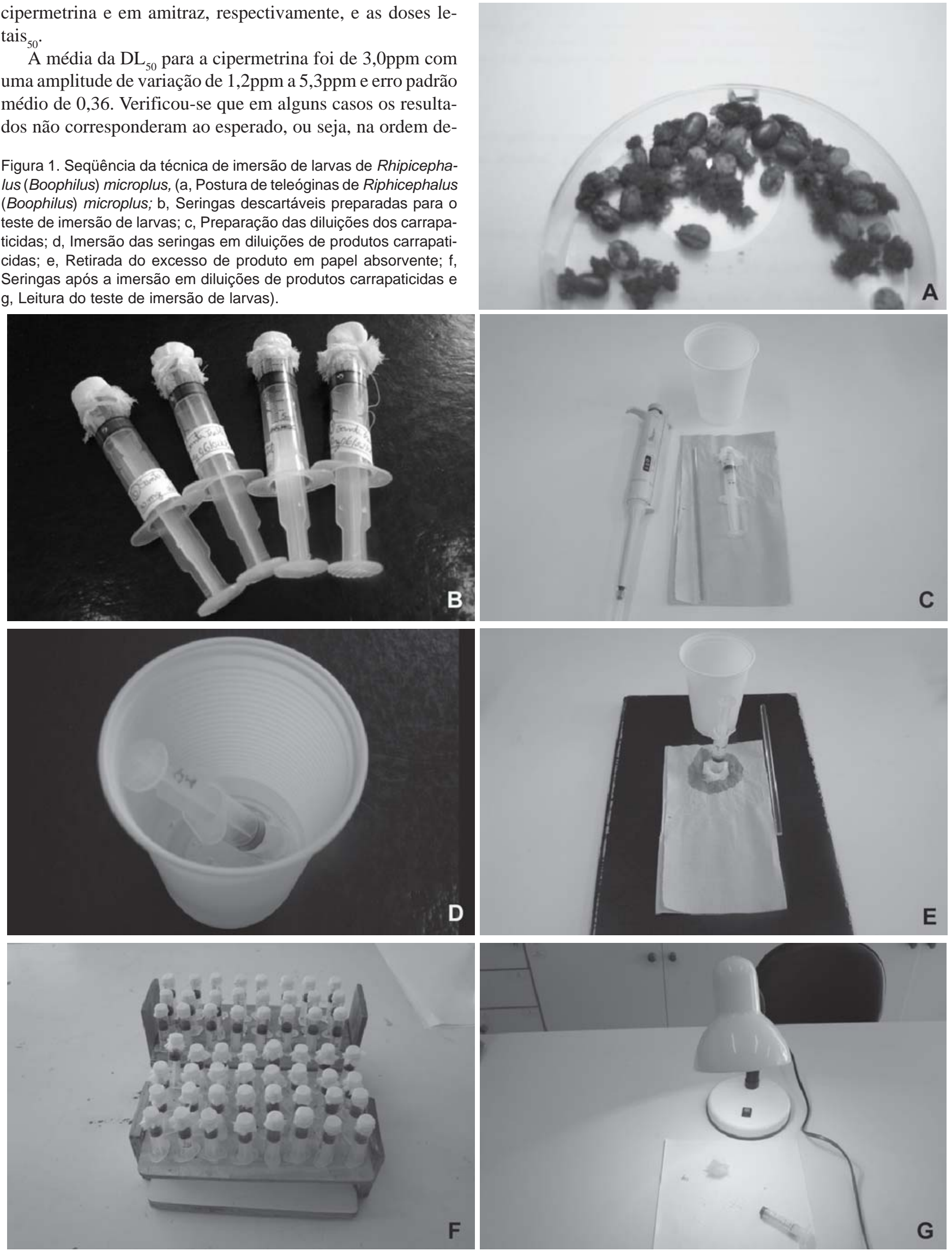
crescente da mortalidade das larvas. Na repetição um, concentração de 37,5ppm, a mortalidade das larvas foi de 63,7\% e na de 18,75ppm, 83,5\%; na repetição sete, na concentração de 18,75ppm, a mortalidade foi de $80,3 \%$ e na de 9,73ppm, foi de $94,2 \%$.

A média da $\mathrm{DL}_{50}$ para o amitraz foi de 17,22ppm com uma amplitude de variação de 3,2ppm a 47,9ppm e erro padrão médio de 4,20. A variabilidade dos resultados foi maior do que com a cipermetrina. Na repetição um, concentração de $62,5 \mathrm{ppm}$, a mortalidade das larvas foi de $76,3 \%$ e na de 31,25ppm, 93,5\%; na repetição dois, na concentração de 15,62ppm, foi de $64,4 \%$ e na de 7,81ppm, 88,1\%; na repetição quatro, a 31,25ppm, foi 76,9\% e na de 15,62ppm, 90,9\%; na repetição nove, a 62,5ppm foi $16,2 \%$ e na de 31,2ppm, $61,4 \%$ e na repetição dez, a $62,5 \mathrm{ppm}$ foi de $70,3 \%$ e na concentração de 31,25ppm, 95,5\%.

A variação dos resultados, com mortalidade maior em concentração menor interfere diretamente na $\mathrm{DL}_{50}$, ficando evidente que nesses casos há necessidade de repetir o teste. Essas variações podem ser explicadas pelo acaso ou pelo erro experimental.

Quando se compara com o teste de imersão de larvas proposto (SHAW, 1966) e com o teste do pacote de larvas (STONE; HAYDOCK, 1962), esta técnica apresenta as vantagens de ser de mais fácil execução, ser realizada em menor tempo e possibilita utilizar produtos comerciais ou princípios ativos puros com maior segurança para o operador.

Com base nos resultados pode-se afirmar que a metodologia descrita para o teste carrapaticida por imersão com larvas apresenta resultados promissores para calcular a média da dose letal ${ }_{50}$ para os acaricidas à base de cipermetrina e amitraz.

\section{REFERÊNCIAS BIBLIOGRÁFICAS}

BARCI, L.G.A.; NOGUEIRA, A.H.C. Método para avaliação de mortalidade de larvas de Boophilus microplus (CANESTRINI, 1887) submetidas a tratamentos com produtos carrapaticidas. Arquivo do Instituto Biológico, v.73, n. 1, p. 105-109, 2006.

MILLER, R.J.; DAVEY, R.B.; GEORGE, J.E. Modification of the Food and Agriculture Organiztion Larval Packet Test to Measure Amitraz-Susceptibility Against Ixodidae. Journal of Medical Entomology, v. 39, n. 4, p. 645-651, 2002.

SABATINI, G.A.; KEMP, D.H.; HUGHES, S; NARI, A.; HANSEN, J. Tests to determine $\mathrm{LC}_{50}$ and discriminating doses for macrocyclic lactones against the cattle tick, Boophilus microplus. Veterinary Parasitology, v. 95, n. 1, p. 53-62, 2001.

SHAW, R. D. Culture of an organophosphorus - resistant strain of Boophilus microplus (Can.) and an assessment of its resistance spectrum. Bulletin Entomological Research, v. 56, n. 3, p. 389-405, 1966.

STONE, B.F.; HAYDOCK, K.P. A method for measuring the acaricide susceptibility of the cattle tick Boophilus microplus. Bulletin of Entomological Research, v. 53, n. 3, p. 563-578, 1962.

VARGAS, M.S.; CÉSPEDES, N.S.; SÁNCHEZ, H.F.; MARTINS, J.R.; CÉSPEDES, C.O.C. Avaliação in vitro de uma cepa de campo de Boophilus microplus (Acari: Ixodidae) resistente à Amitraz. Ciência Rural, v. 33, n. 4, p. 737-742, 2003.

Recebido em 19 de fevereiro de 2008.

Aceito para publicação em 10 de novembro de 2008. 